\title{
A Cost Effective Solution to Security Constrained Unit Commitment and Dispatch Problem using Hybrid Search Algorithm
}

\author{
Sushil Prashar ${ }^{1}$, Vikram Kumar Kamboj ${ }^{2, *}$, Kultaar Deep Singh $^{3}$ \\ ${ }^{1}$ Ph.D Research Scholar, School of Electronics and Electrical Engineering, Lovely Professional University, Punjab, INDIA \\ ${ }^{2}$ Domain of Power Systems, School of Electronics and Electrical Engineering, Lovely Professional University, Punjab, INDIA \\ ${ }^{3}$ Departmement of Electrical Engineering, Shaheed Bhagat Singh State Technical Campus, Ferozepur, Punjab, INDIA
}

\begin{abstract}
The Security constraints unit commitment (SCUC) is a powerful scheduling technique used in power markets for daily planning. SCUC is a framework that combines two common algorithms in the electricity industry: Unit Commitment (UC) and Economic Dispatch (ED), while adding a new dimension Security. Load demand is changing continuously due to variation of load of industrial, residential and commercial sectors. Thus, it is very important to decide which generating unit should be kept on and how much power should be dispatched so that time varying load demand can be meet on Hourly basis and there should be no scope of occurrence of loss of load hour (LOLH). In the proposed research, the recently developed hybrid meta-heuristics search algorithm i.e. GWO-RES has been applied to solve the security constrained Unit Commitment and dispatch problem of Electric power system. The efficiency of the proposed hybrid algorithm has been tested for standard IEEE-14 Bus, 30-Bus and 56-bus system and it has been experimentally found that GWO-RES performs much better than hybrid GWO-PS and GWO-RS algorithm.
\end{abstract}

Keywords: IEEE-14 Bus, IEEE-30 Bus, IEEE-56 Bus, Security Constrained Unit Commitment Problem.

\section{Introduction}

Security Constrained unit commitment and dispatch effort pair in power generation industry to empower grid management and power generation, along with ensuring proper dispatch of power on hourly basis. Now a days, in power sector, there are different kinds of electric power generating stations like nuclear, thermal and hydro power plants etc. During a day, the demand of electric power is changing continuously and achieves various peak values. In this way, it is essential to choose which power generation units have to turn on and in which hour it is required in the power system framework arrange and furthermore, for what time period the generating unit should keep in shut down condition to main the cost viability of turning on and closing down of particular unit. The whole procedure of making and computing on these choices is recognized as unit commitment (UC). The power generating unit that is planned for association with network of electrical power sector is known as the committed unit [1]. In power systems, the unit commitment problems refers such kind of problem which are associated with deciding the off/on conditions of producing units to limit the working expense for a specified time period. Power generators can't be directly turn on to fulfil up load demand of the electric power. So it is necessitated that the arranging of producing units is to be done in such a way, that there is sufficient amount of power generation accessible to satisfy the demand of the electrical load along with maintain malfunctions and failures under adverse conditions. Unit responsibility handle the power generation plan for electrical system for minimize fuel and operational costs while fulfilling system and constraints, for example, demand of electrical load and system save necessities over a set of time spans. The Security constraints unit commitment problem (SCUCP) is a powerful scheduling technique used in power markets for daily planning. SCUC is a framework that combines two common algorithms in the electricity industry: Unit Commitment (UC) and Economic Dispatch (ED), while adding a new dimension - Security [2].

SCUCP is fundamentally about discovery the most appropriate timetable to turn-off or turn-on the power units to fulfil the electrical power need for a while and simultaneously keep the expense of power generation however much least as could be expected. SCUCP contains a large scale, nonlinear and mixed integer constrained optimization which to have a place with combinatory improvement issues. Here are numerous

* Corresponding author: vikram.23687@lpu.co.in 
constraints included with SCUCP and consequently it is a tedious and complex process for computation and find out the optimum solution for SCUCP [3]. The SCUCP is to decide a small amount of price turn-off and turn-on arrangement for set of power generation units to fulfil an electricity need although fulfilling system physical and operational requirements connected with different power generation units. The price for start-up, shutdown, no load cost and fuel are included in the cost for production. The operational constraints which must be thought about include (I) the total amount of electric power generation should achieve the electricity demand along with losses to that generating system, (II) there should be sufficient spinning reserve to withstand with any shortfall in electricity generations, (III) the generation limit of each generator units must be within limit of its maximum and minimum capacity and (IV) minimum down time and minimum up time of each and every power generating unit should be pragmatic. The unit commitment is intended to figure out with an appropriate generator commitment schedule for electric power sector over a time horizon of one day to a week. The fundamental objective of SCUCP is to minimize the total generation cost by maintaining LOLH and satisfying some physical and system constrains forced on the system, for example, operating constraint, load-power generation balance, spinning reserve, minimum down time horizon and minimum up time horizon and initial status of such generating unit and so on [4]. A few ordinary techniques are accessible to take care of the SCUCP. The premier target of SCUCP is to locate the optimum scheduling for working of the accessible generating units to regulate the total cost of power generation and along with total cost for operation of that power generating unit. The complete cost of electric power generation incorporates with the cost of shut down of the unit, cost of fuel and the cost of start-up of the unit. The cost of fuel are reliant on price of the fuel the qualities information of power generating units, for example, heat pace of producing units, turn-on and off schedule and initial status of the power generation unit. So, the main objective of security constrained unit commitment problem is to minimize the total operating cost with satisfying those constrains.

\section{Problem Formulation}

The generating power is distributed along with utilities of generator scheduling which will meet the time varying load demand for a specific time period is known as Unit Commitment Problem (UCP). The actual objectives of UCP is minimization of overall cost for production considering different system constraints. The overall cost of production including sum of shutdown cost \& start-up cost, cost of fuel are given below:

$$
\min (T F C)=\sum_{h=1}^{H} \sum_{i=1}^{N G}\left\{F_{\cosh i}\left(P_{h i}\right)+S U C_{h i}+S D C_{h i}\right\}
$$

The total cost of fuel over the scheduled time span ' $h$ ',

$T F C=\sum_{h=1}^{H} \sum_{i=1}^{N G}\left[F_{\cosh i} \times U_{h i}+S U C_{h i}\left(1-U_{i,(h-1)}\right) \times \mathrm{U}_{h i}\right]$
$T F C=\sum_{h=1}^{H} \sum_{i=1}^{N G}\left[\left(A_{i} P_{i}^{2}+B_{i} P_{i}+C_{i}\right) \times U_{i, h}+S U C_{i, h}\left(1-U_{i,(h-1)}\right) \times \mathrm{U}_{i, h}\right]$

$F_{\cosh i}\left(P_{h i}\right)=$ Cost of Fuel for a particular generating unit $\mathrm{i}^{\text {th }}$ at that particular time ' $h$ ' hour

$S U C_{i h}=$ Cost of Start-up for $i^{\text {th }}$ unit within ' $h$ ' hours

$S D C_{i h}=$ Cost of Shutdown for $\mathrm{i}^{\text {th }}$ unit within 'h' hours

$P_{h i}^{M A X}=$ Maximum electrical power generation by unit $\mathrm{i}$

$P_{i}^{M I N}=$ Minimum electrical power which generation by unit $\mathrm{i}$

$P_{i, h}=$ Electrical power generation of unit $\mathrm{i}^{\text {th }}$ at the time span' $h$ '

$P D_{h}=$ Load Demand at' $h$ ' hours

$I N S_{i}=$ Initial status of unit $\mathrm{n}$ at time' $\mathrm{h}$ '

$T_{i, h}^{\text {OFF }}=$ Initial OFF status for $\mathrm{nth}$ unit at time' $\mathrm{h}$ '

$T_{i, h}^{O N}=$ Initial ON status for nth unit at time' $h$ '

$T_{i}^{U P}=\mathrm{UP}$ condition for i no. of power generating unit

$T_{i}^{D W}=$ DOWN condition for i no. of power generating unit

$S R_{h}=$ Spinning reserve necessity

Here, cost for fuel $F_{\text {cosih }}\left(P_{i h}\right)$ is stated as quadratic design that mostly working by researchers, also named as equation of convex function.

For the cost of fuel of (n) unit at ( $t$ ) hour can be mathematically represented as such an equation which is given below:

$F_{\text {cosih }}\left(P_{i}\right)=A_{i} P_{i}^{2}+B_{i} P_{i}+C_{i}$

Where $A_{i} \quad B_{i}$ and $C_{i}$ are represented as coefficients of cost that may expressed as $\$ / h, \$ / M W h$ and $\$ / M W h^{2}$ correspondingly.

Start-up cost can mathematically represented by step function which is given below:

$S U C_{i h}=\left\{\begin{array}{lll}H S U_{i} ; & \text { for } & T_{i}^{D W} \leq T_{i}^{U P} \leq\left(T_{i}^{D W}+T_{i}^{C O L D}\right) \\ C S U_{i} ; & \text { for } & T_{i}^{U P}>\left(T_{i}^{D W}+T_{i}^{C O L D}\right)\end{array}\right.$

In usual value of the Shutdown cost for standard system is denoted as zero and this can be established as fixed cost followed by the eqn. (5).

$$
\mathrm{SDC}_{i h}=\mathrm{KP}_{i h}
$$

Where $\mathrm{K}$ is represented as incremental cost for shutdown.

Which is subjected through some constraints followed by: (1) System constraints and (2) Unit constraints

System Constraints:

System constrains are interrelated with all generating unit existing in the systems. The systems constrains are characterised into two types like:

Power Balance or Load Balance Constraints 
In power system the constraint including power balance or load balance is more important parameter consist of summation of whole committed generating unit at $t^{\text {th }}$ time span must be larger than or equivalent to the power demand for the particular time span ' $\mathrm{t}$ '

$$
\sum_{i=1}^{N U} P_{i, h} \times U_{i, h}=P D_{i}
$$

\section{Spinning Reserve (SR) Constraints}

Reliability of the system can be considered as facility of extra capability of power generation that is more important to deed instantly when failure is occurred due to sudden change in load demand for such power generating unit which is already running. The extra capability of power generation is recognized as Spinning Reserve which is exactly represented as:

$$
\sum_{i=1}^{N U} P_{i, h}^{M A X} \times U_{i, h} \geq P D_{h}+S R_{h}
$$

Step 1: Sort the generators in descending order of maximum generating capacity Step2: for $\mathrm{g}=1$ to $\mathrm{G}$

$$
\text { if } u_{g, h}=0
$$

$$
\begin{aligned}
& \text { then } u_{g, h}=1 \\
& \text { else if } T_{g, h}^{O F F}>M D T_{g} \\
& \text { then } T_{g, ~}^{O N}=T_{g, h-1}^{O N}+1 \\
& \text { and } T_{g, h}^{O F F}=0
\end{aligned}
$$

Step-3: Verify new generating power of units.

$$
\text { Step-4: if } \sum_{j=1}^{N G} P_{\mathrm{j} \max } u_{j, h} \geq D_{h}+R_{h} \text { then stop the algorithm, else go to step-2. }
$$$$
\text { Step-5: if } T_{O F F}^{g, h}<M D T_{g} \text { then do } l=h-T_{g, h}^{O F F}+1 \text { and set } u_{g, h}=1
$$$$
\text { Step-6: Calculate } T_{g}^{l}=T_{g,-1}^{O N}+1 \text { and } T_{g, t}^{\text {OFF }}=0
$$$$
\text { Step-7: if } 1 \gg \mathrm{h} \text {, Verify generator output power for } \sum_{j=1}^{N} P_{\mathrm{j} m \mathrm{max}} u_{j, h} \geq P D_{i}+S R_{i} \text {, else }
$$
increment 1 by 1 and go to step- 5

Fig. 1(a). PSEUDO code of SR repairing in SCUCP

Constraints for Power Generating Unit

The specific constraints related with particular power generating unit exist in the systems are called generating unit constraint which are given as:

Thermal unit constraints

Thermal power units are controlled manually. This types of unit need to undertake the change of temperature gradually. So it take certain time span to take the generating unit accessible. So some crew members are essential to execute the maintenance and procedure of some thermal power generating units.

Minimum up Time

This constraint is defined as here will be minimum period of time previously the unit can be start over when the unit have already been shut down which is mathematically defined as:

$$
T_{i, h}^{O N} \geq T_{i}^{U P}
$$

Where, $T_{i, h}^{O N}$ is defined as interval through which the generating unit $\mathrm{i}$ is constantly $\mathrm{ON}$ (in hours) and $T_{i}^{U P}$ is defined as minimum up time (in hours) for the generating unit $\mathrm{n}$.

Minimum down Time

When the power generating units will be DE-committed, there is required least period of time for recommitted of the unit which is mathematically given as:

$$
T_{i, h}^{O F F} \geq T_{i}^{D W}
$$

Where, $T_{i, h}^{O F F}$ is time period for which generating unit $\mathrm{n}$ is constantly OFF (in hrs) and $T_{i}^{D W}$ is denoted as minimum down time (in hours) for the unit.

To adequate minimum downtime and up time repair by heuristic mechanism is accepted those stages are stated as below in Fig. 1(b).

$$
\begin{aligned}
& \text { for } \begin{array}{l}
h=1 \text { to } \mathrm{H} \\
\text { if } \mathrm{h}==1 \\
\text { Compute } T_{h}^{O N}=T_{h}^{O N} U_{h i}+U_{h i}{ }^{\prime} \\
\text { Compute } T_{h}^{O F F}=\left(T_{h}^{O F F}\right)^{\prime} \bar{T}_{h}^{O N}+\bar{T}_{h}^{O N} \\
\text { else } \\
\text { Compute } T_{h}^{O N}=T_{h-1}^{O N} U_{h i}{ }^{\prime}+U_{h i}{ }^{\prime} \\
\text { Compute } T_{h}^{O F F}=T_{h-1}^{O F F} \bar{T}_{h}^{O N}+\bar{T}_{h}^{O N}
\end{array} \\
& \text { end }
\end{aligned}
$$

Fig. 1(b). PSEUDO code for MUD/MUT constraints

Max and Min Electric Power Generating Limits All electricity generating unit have its individual max/ min electric power generating limit, below and outside which will cannot produce and this is known as maximum and minimum power limits, which is mathematically written as:

$$
P_{i}^{M I N} \leq P_{i, h} \leq P_{i}^{M A X}
$$

Initial Status for operation of electrical units

For every units have initial operating position that must proceeds as the day's earlier generation scheduled are taken into consideration, thus each and all generating units can fulfils its lowest down/up time.

Crew Constraint

When any power plant consist of added one units and they couldn't turn on at the same time period. So here need more than one crew members to attend such units in a same time while starting up.

Unit Accessibility Constraint

For the constraint shows accessibility of power generating unit surrounded by any of the resulting various circumstances:

A) Accessible or Non Accessible 
B) Must Outage or Out

C) Must running condition

Initial Status of Electricity Generation Unit

It signifies value of initial grade of power generating unit. Its favourable rate signifies the position of current generating unit which already in up condition, which mean about numeral time periods of the generating units are previously up, and for its negative value which is an index of the integer of hour then power generating unit has been previously in down condition. For the position of generating unit $+/$ - earlier the 1 st hour through the schedule which is an essential feature to define where its newest situation interrupts the constraint of $T_{i}^{U P} \& T_{i}^{D W}$. LOLH is properly taken into consideration while solving SCUC Problem.

\section{Hybrid GWO-RES Algorithm}

In the proposed research, the recently developed grey wolf optimizer [5] has been hybridized with Random exploratory search algorithm to improve the exploitation phase of the existing GWO algorithm. The mathematical formulation for the hybrid GWO-RES algorithm can be found in [6] and PSEUDO code for the proposed optimizer has been shown in Fig.2.

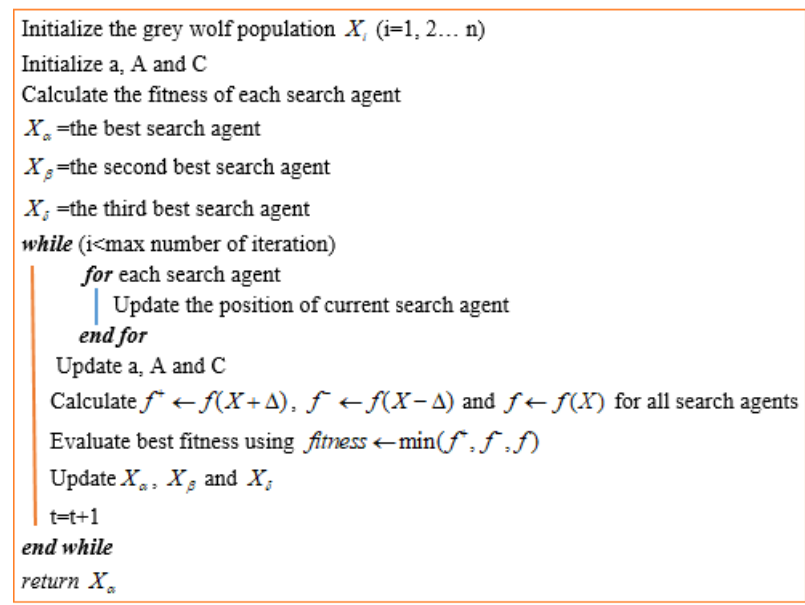

Fig. 2. PSEUDO code for proposed GWO-RES algorithm

\section{Results \& Discussions}

The recently developed GWO-RES algorithm has been applied to solve security constrained unit commitment problem of electric power system. In order to validate the results using GWO-RES algorithm, IEEE-14 Bus, IEEE-30 Bus and IEEE-56 Bus system along with 10generating units system has been taken into consideration [7] and Generation Schedule of Committed Units for IEEE-14 Bus system is shown in Fig.3, Generation schedule for IEEE-30 bus system has been shown in Fig.4, generation schedule of committed power generating units for IEEE-56 bus system has been shown in Fig.5 and test results for 10-Unit Test System at 10\%
Spinning Reserve are shown in Fig.6 and Table-1 and Table-2.

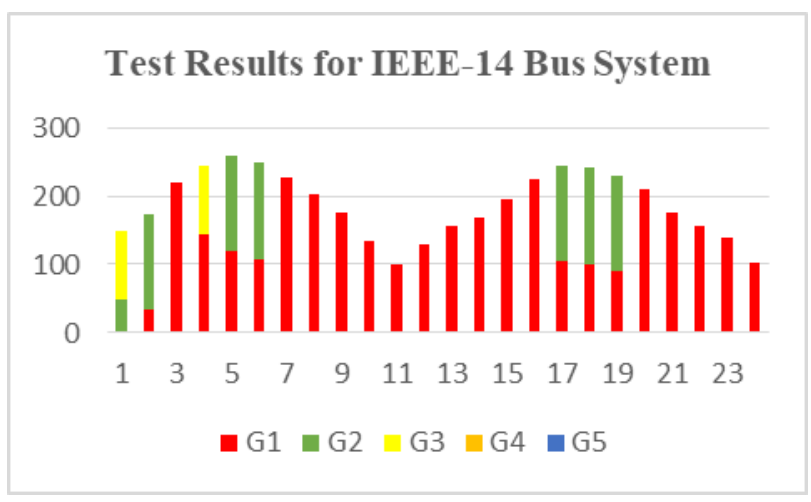

Fig. 3. Hourly dispatch of Committed Units for IEEE-14 Bus System

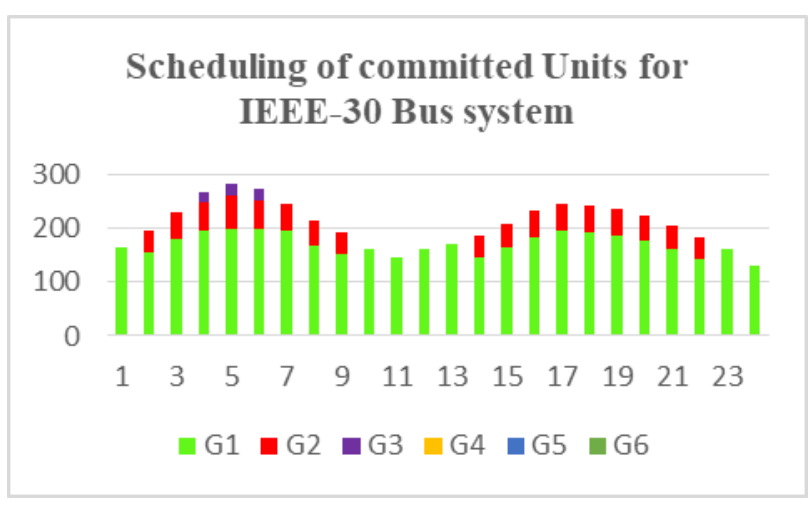

Fig. 4. Hourly dispatch of Generation Schedule of Committed Units for IEEE-30 Bus System

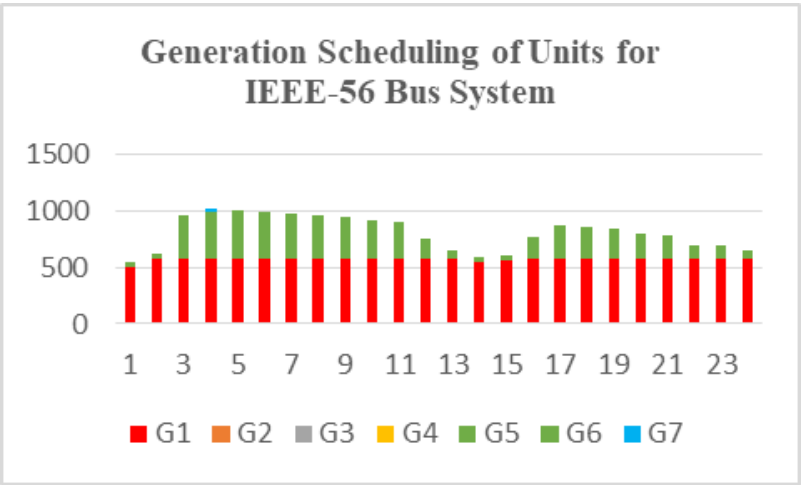

Fig. 5. Hourly dispatch of Committed Units for IEEE-56 Bus System

Table 1. Results of SCUCP for 10 -unit system at $10 \%$ SR

\begin{tabular}{|c|c|c|c|c|}
\hline Method & Best Cost & $\begin{array}{c}\text { Average } \\
\text { Cost }\end{array}$ & $\begin{array}{c}\text { Worst } \\
\text { Cost }\end{array}$ & Best Time \\
\hline $\begin{array}{c}\text { GWO- } \\
\text { RES }\end{array}$ & 563977.017 & 564078.2 & 564388.78 & 134.810935 \\
\hline
\end{tabular}


Table 2. Alpha, Beta and Delta Score Obtained through GWORES

\begin{tabular}{|c|c|c|c|}
\hline Method & Alpha Score & Beta Score & Delta Score \\
\hline GWO-RES & 563977.017 & 564018.158 & 564588.965 \\
\hline
\end{tabular}

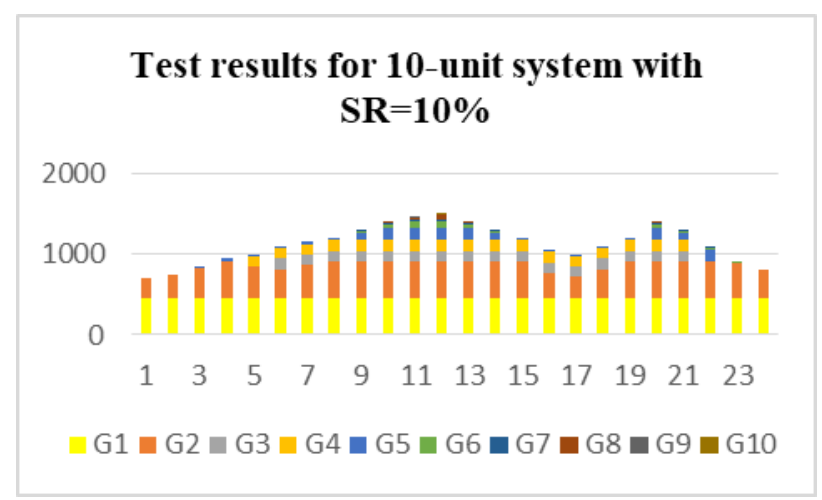

Fig. 6. Hourly dispatch of Committed Units for 10-unit System

\section{CONCLUSIONS}

In the suggested research work, the authors has successfully presented the solution of security constraints unit commitment problem using hybrid metaheuristics search algorithm. The proposed GWO-RES algorithm was developed to enhance the exploitation phase of the existing grey wolf optimizer and it has been successfully tested for IEEE-14 Bus, IEEE-30 Bus, IEEE-56 Bus and 10-generating units of electric power system.

\section{References}

1. V. Kumar, S. K. Bath, and J. S. Dhillon, "Electrical Power and Energy Systems Implementation of hybrid harmony search / random search algorithm for single area unit commitment problem," Int. J. Electr. POWER ENERGY Syst., vol. 77, pp. 228249, 2016.

2. H. Park, Y. Gyu, and J. Park, "Electrical Power and Energy Systems Stochastic security-constrained unit commitment with wind power generation based on dynamic line rating," Electr. Power Energy Syst., vol. 102, no. April, pp. 211-222, 2018.

3. M. Reza Norouzi, A. Ahmadi, A. Esmaeel Nezhad, and A. Ghaedi, "Mixed integer programming of multi-objective security-constrained hydro/thermal unit commitment," Renew. Sustain. Energy Rev., vol. 29, pp. 911-923, 2014.

4. E. Nasrolahpour and H. Ghasemi, "A stochastic security constrained unit commitment model for reconfigurable networks with high wind power penetration," Electr. Power Syst. Res., 2014.

5. S. Mirjalili, S. M. Mirjalili, and A. Lewis, "Grey Wolf Optimizer," Adv Eng Softw, vol. 69, p. 46, 2014.
6. A. Bhadoria and V. K. Kamboj, "Optimal generation scheduling and dispatch of thermal generating units considering impact of wind penetration using hGWO-RES algorithm," Appl. Intell., 2018.

7. V. K. Kamboj, "A novel hybrid PSO - GWO approach for unit commitment problem," Neural Comput. Appl., 2015. 